\title{
Electric field tuning of magnetization in an antiferroelectric-based heterostructure: Example of NMG/PLZST/NMG
}

\author{
Liuyang Han ${ }^{1,2,3}$, Freddy Ponchel ${ }^{2}$, Nicolas Tiercelin ${ }^{2}$, Denis Rémiens ${ }^{2}$, Tuami Lasri ${ }^{2}$, Philippe \\ Pernod $^{2} \&$ Genshui Wang ${ }^{3}$
}

1-Key Laboratory of Inorganic Functional Materials and Devices and State Key Laboratory of High Performance Ceramics and Superfine Microstructure, Shanghai Institute of Ceramics, Chinese Academy of Sciences, Shanghai, China

2-Univ. Lille, CNRS, Centrale Lille, ISEN, Univ. Polytechnique Hauts-de-France, UMR 8520-IEMN, Joint International Laboratory LIA LICS/LEMAC, Lille, France

3-University of Chinese Academy of Sciences, Beijing, China

\begin{abstract}
A magnetoelectric (ME) heterostructure was fabricated by depositing a Ni-Mn-Ga (NMG) magnetic film on an antiferroelectric (AFE) PLZST- $(\mathrm{Pb}, \mathrm{La})(\mathrm{Zr}, \mathrm{Sn}, \mathrm{Ti}) \mathrm{O}_{3}$-ceramic substrate. Significant and reversible electric field-induced relative magnetization changes $\left(\Delta M / M_{0}\right)$ were firstly observed in this magnetic/AFE heterostructure, and the $\Delta M / M_{0}$ vs electric field loops have been found to correspond well with the strain-electric field curve of PLZST ceramic. The maximum $\Delta M / M_{0}$ variation can reach $15 \%$, indicating an obvious strain-mediated converse magnetoelectric (CME) effect. A maximum CME coefficient of $1.14 \times 10^{-9} \mathrm{~s} / \mathrm{m}$ was obtained at the switching electric fields of PLZST. The collected results suggest that AFE materials with a large electric-field-induced phase transition strain could be alternative candidates in $\mathrm{ME}$ coupling heterostructures.
\end{abstract}

\section{Introduction}

The coexistence of ferroelectricity and ferromagnetism in magnetoelectric (ME) materials has given rise to advanced multifunctional properties for the development of novel devices in a large range of applications [1-3]. Particularly, the electric field tuning of magnetism, known as converse magnetoelectric (CME) effect, has drawn ever-increasing interest for potential applications in information storage, tunable microwave devices, and spintronic devices [4-6]. Since single phase ME materials (or intrinsic multiferroic compounds, such as $\mathrm{BiFeO}_{3}$ and $\mathrm{BiMnO}_{3}$ ) are rare and their $\mathrm{ME}$ effect is weak and usually far below room temperature $[7,8]$, multiferroic composites with combined ferromagnetic (FM) and ferroelectric (FE) phases have been exploited to electrically control magnetic order $[9,10]$.

Among all the coupling mechanisms, the strain-mediated CME coupling, where the transfer of strain from a FE material to a FM material is the origin of the CME effect, has been mostly used. Much advancement has recently been achieved, but most of previous works are focused on FM/FE heterostructures, such as $\mathrm{CoFe}_{2} \mathrm{O}_{4} / \mathrm{BaTiO}_{3}, \mathrm{Ni}_{0.8} \mathrm{Zn}_{0.2} \mathrm{Fe}_{2} \mathrm{O}_{4} / \mathrm{Pb}(\mathrm{Zr}, \mathrm{Ti}) \mathrm{O}_{3}$, Terfenol$\mathrm{D} / \mathrm{Pb}(\mathrm{Mn}, \mathrm{Nb}) \mathrm{O}_{3}-\mathrm{PbTiO}_{3}, \mathrm{FeBSiC} / \mathrm{PVDF}$ and so on [11-14]. AFE materials, with novel properties of electric-field-induced AFE/FE phase transition, exhibit a large and sharp strain change during the phase transition for atomic rearrangements and zero polarization and strain in relaxed state. On the one hand, the significant strain of AFEs, which can reach $0.4 \%$ in some AFE ceramics, make them extremely attractive for strain-mediated ME coupling[15]. On the other hand, the strain of FEs always peaks at $E_{\mathrm{C}}$, where the peak is so sharpened that only a very small and limited voltage range is available to obtain a large strain[16] Concerning AFEs, a large strain can be obtained once the electric field is higher than the switching field, which 
provides large practical operating voltage range for strains. So, the distinct characteristics of AFE materials give an additional opportunity to the electric field tuning of magnetism. However, very few works are dedicated to ME coupling of FM/AFE heterostructures, which seems to be interesting and promising in multiferroic composites.

In this work, the PLZST ceramic in a composition of $\mathrm{Pb}_{0.97} \mathrm{La}_{0.02} \mathrm{Zr}_{0.60} \mathrm{Sn}_{0.30} \mathrm{Ti}_{0.10} \mathrm{O}_{3}$ was chosen as the AFE substrate for its high electric-field-induced strain. The Ni-Mn-Ga (NMG) film was considered for the FM phase as well as the electrode because of its strong combination of magnetic and elastic orderings and good conductivity[17]. The NMG/PLZST/NMG multiferroic heterostructure was fabricated to demonstrate electric field control of magnetization of FM/AFE/FM laminates at room temperature. By introducing electric fieldinduced AFE-FE phase transition of PLZST substrate into the magnetoelectric system, an obvious converse ME effect has been obtained. The relative magnetization changes in the heterostructure vs the electric field, $\left(\Delta M / M_{0}\right)$-Ecurves, show comparable evolutions to those of strain-electric field $(S-E)$ curves of AFE substrate, indicating that FM/AFE multiferroic heterostructures can be a good design for strain-mediated ME coupling effect.

\section{Experiments}

The PLZST ceramics were prepared by a conventional solid-state reaction process. $\mathrm{Pb}_{3} \mathrm{O}_{4}$, $\mathrm{La}_{2} \mathrm{O}_{3}, \mathrm{ZrO}_{2}, \mathrm{SnO}_{2}$, and $\mathrm{TiO}_{2}$ powders (chemical purities $\geq 99.0 \%$ ), with 0.5 wt. $\%$ excess $\mathrm{Pb}_{3} \mathrm{O}_{4}$ to compensate $\mathrm{Pb}$ volatilization during sintering were used. The sintered PLZST ceramic was machined and fine polished into $10 \times 10 \times 0.5 \mathrm{~mm}^{3}$ substrates. Then the NMG films $(700 \mathrm{~nm}$ thick) were deposited on the two sides of PLZST substrates by radio-frequency magnetron sputtering at room temperature with a nominal alloy-composition target of $\mathrm{Ni}_{0.5} \mathrm{Mn}_{0.33} \mathrm{Ga}_{0.17}$. The details are given in the reference [18]. The samples X-ray diffraction (XRD) patterns were performed using a Rigaku D X-ray diffractometer with a $\mathrm{Cu} K \alpha$ radiation. The scanning electron microscopy (SEM) was employed to characterize the interface of NMG film and PLZST ceramic. The electric hysteresis loop $(P-E)$ and field-induced strain curve $(S-E)$ of the PLZST are obtained with a standard ferroelectric tester (TF analyzer 2000, AixACCT, Germany). The in-plane magnetic hysteresis loops $(M-H)$ are measured at room temperature using a Vibration Sample Magnetometer (VSM, ADE model EV9) with a vibration frequency of $75 \mathrm{~Hz}[19]$. The NMG film is isotropic so the direction of the magnetic field does not matter and can be indifferently along the $x$ or $y$ axis (Figure 1D). 

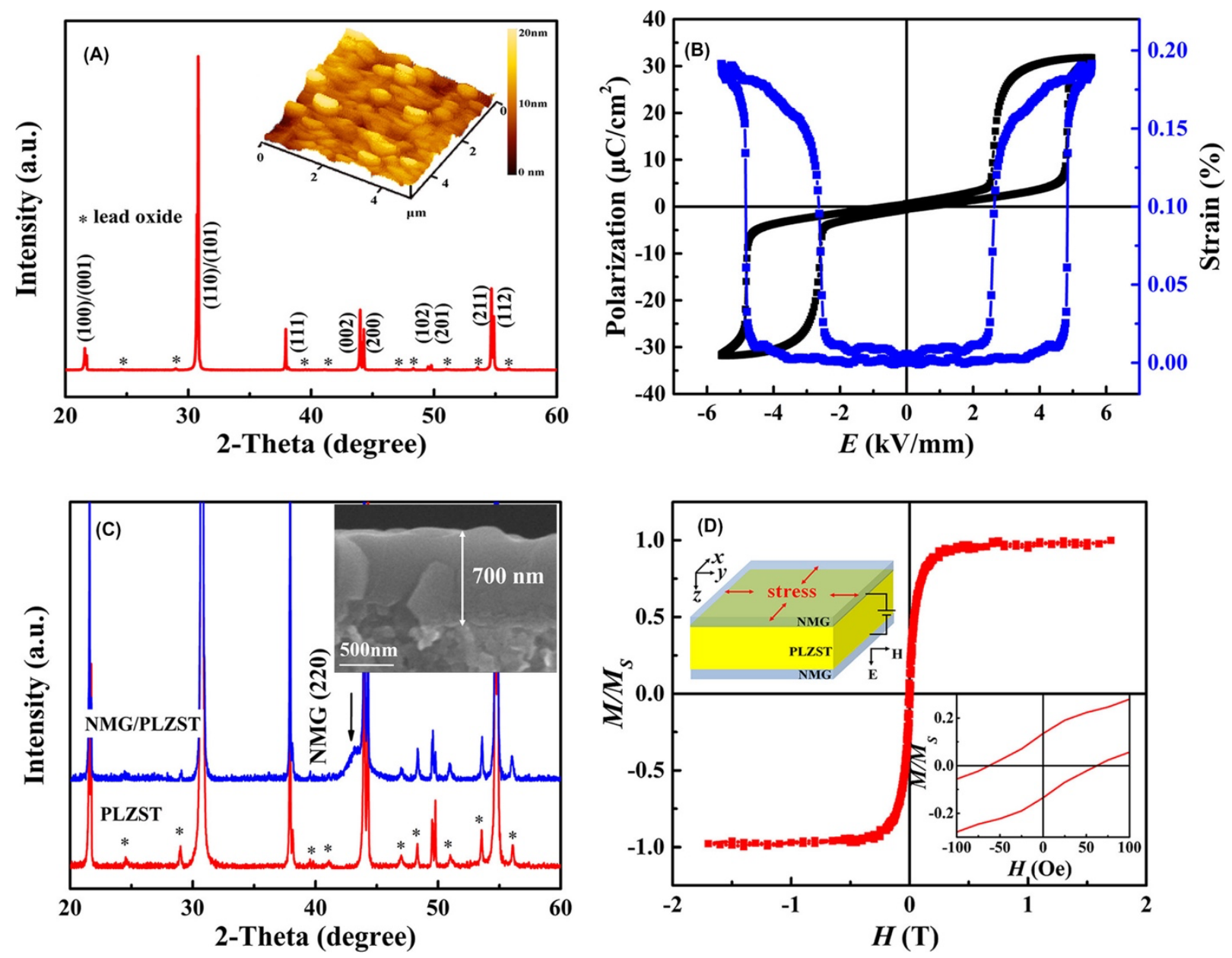

Figure 1: A, XRD pattern and inset AFM image of PLZST; B, $P-E$ and $S-E$ loops of PLZST; C, XRD patterns of PLZST, NMG/PLZST samples, inset the cross-sectional SEM picture of NMG/PLZST and $\mathrm{D},(M-H)$ loops, inset the schematic and magnified $(M-H)$ loops of NMG/PLZST/NMG sample.

The electric field tuning of magnetization $(M-E)$ is performed by means of the VSM with an external DC electric field (High voltage supply, SRS Model PS350) applied to the sample via two coppers attached to the upper and lower conductive NMG films. For one specific electric field applied to the sample, the dwell time is three minutes for the stabilization of magnetic response and recording of averaged data.

\section{Results and discussion}

The XRD pattern of PLSZT ceramic is given in Figure 1A, and the atomic force microscopy (AFM) image of the PLZST substrate surface is inset. The PLZST substrate is well-crystallized into tetragonal AFE phase with small traces of lead oxide phase, which is due to the lead excess in the ceramic preparation process. Since the substrate roughness plays an important role in film growth and coupling effect, the PLZST ceramic is well polished with a resulting average roughness of $\sim 10 \mathrm{~nm}$. The $P-E$ loop and $S-E$ curve given in Figure $1 \mathrm{~B}$ show a typical AFE characteristic. The forward and backward switching fields of PLZST are $5 \mathrm{kV} / \mathrm{mm}$ and $2.5 \mathrm{kV} / \mathrm{mm}$, respectively. Once the electric field-induced phase transition between tetragonal AFE phase and rhombohedral FE phase happens, a large strain change is generated simultaneously. The maximum strain value along out-of-plane direction can reach $0.2 \%$, whereas a large strain along in-plane can also be expected which provides opportunities for strain-mediated CME coupling effect. 
As the multiferroic composite interface quality plays a key role on the magnetoelectric coupling [20] the PLZST/NMG interface has been checked. The interface is clear, indicating no significant diffusion between the film and the ceramic substrate. The XRD patterns of NMG films on well-polished PLZST ceramic and $\mathrm{Si} / \mathrm{SiO}_{2}$ substrates are given in Figure 1C. The presence of Bragg peak $\left(2 \theta \sim 43^{\circ}\right)$ in NMG/PLZST which can be indexed as (220) cubic setting corresponds to the austenitic phase of $\mathrm{Ni}_{2} \mathrm{Mn}_{0.85} \mathrm{Ga}_{0.85}$, as we have reported before[18]. A typical magnetic hysteresis $(M-H)$ loop is observed for NMG/PLZST/NMG, and the $H_{\mathrm{C}}$ is around 65 Oe, as shown in Figure 1D.

The magnetization changes vs electric field $(M-E)$ were carried out. In order to characterize it, the magnetization difference $(\Delta M)$ is defined as $\Delta M=M_{\mathrm{E}}-M_{0}$, where $M_{\mathrm{E}}$ is the magnetization for $E$ and $M_{0}$ is the magnetization without electric field. Thus, several relative magnetization changes $\left(\Delta M / M_{0}\right)$ vs $E$ curves are illustrated in Figure $2 \mathrm{~A}$ as representatives. The $\left(\Delta M / M_{0}\right)-E$ loops are symmetric with positive and negative $E$ (as confirmed by Figure $3 \mathrm{~A}$ ), thus only the $\left(\Delta M / M_{0}\right)-E$ curves under positive voltages are given. All the curves show a similar horn-like shape, and sharp reductions of $\Delta M / M_{0}$ happening at the switching field of PLZST. It is necessary to note that the maximum of $\Delta M / M_{0}(15 \%)$ is reached at $0 \mathrm{Oe}$, which is a very important asset in terms of design simplification and cost reduction, thus suggesting significant potential for future magnetoelectric devices. To better reveal the relationship between strain and magnetization change, the absolute value of $\Delta M / M_{0}$ vs $E\left(\left|\Delta M / M_{0}\right|-E\right)$ curve and $(S-E)$ loop of PLZST are given together in Figure 2B for 500 Oe. The $\left|\Delta M / M_{0}\right|-E$ curve is in good agreement with the $(S-E)$ loop of PLZST. Once a large strain is generated in the PLZST substrate upon application of the electric field, the magnetization of NMG films greatly decreases, indicating a strong converse ME coupling effect. In theory, the field-induced strain originating from the field-induced AFE-FE phase transition deformation of the PLZST ceramic leads to anisotropy change in NMG films. In this case, the PLZST strain changes the magnetic domain configuration, which leads to a magnetization reduction and the sign of $\Delta M / M_{0}$ is negative. The strain destroys the alignment of well-ordered magnetic domains and favors to rotate magnetic moments toward opposite direction. The high agreement of $S-E$ and $\left|\Delta M / M_{0}\right|-$ $E$ evolution demonstrates that AFE materials with strong electric field-induced phase transition strain show great potential in ME coupling effect and can be promising candidates for multiferroic heterostructures. 

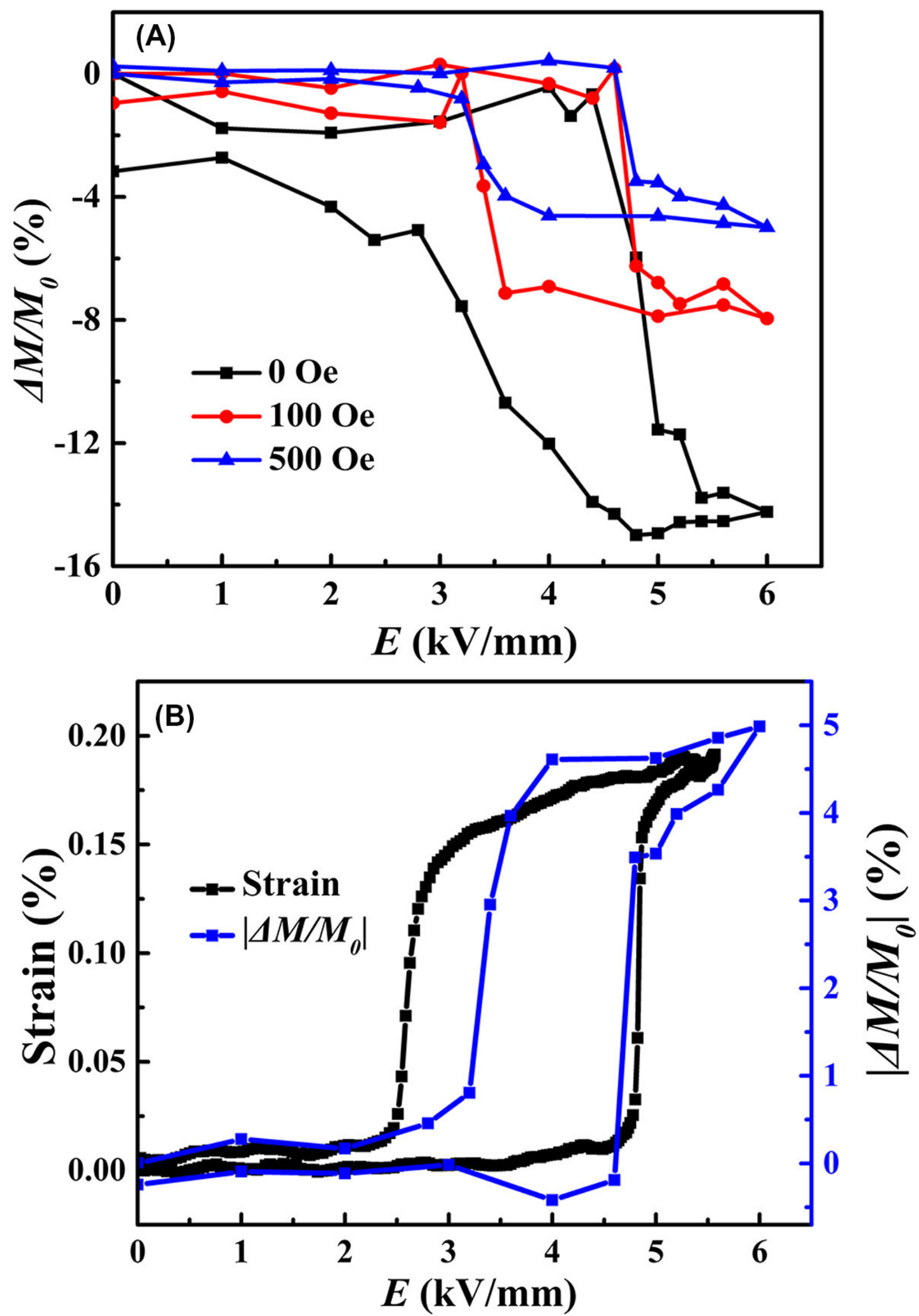

Figure 2: $\mathrm{A},\left(\Delta M / M_{0}\right)-E$ curves under bias magnetic field of 0,100 , and $500 \mathrm{Oe}$ and $\mathrm{B},(S-E)$ loop and $\left|\Delta M / M_{0}\right|-E$ loop measured at 500 Oe of NMG/PLZST/NMG sample. 

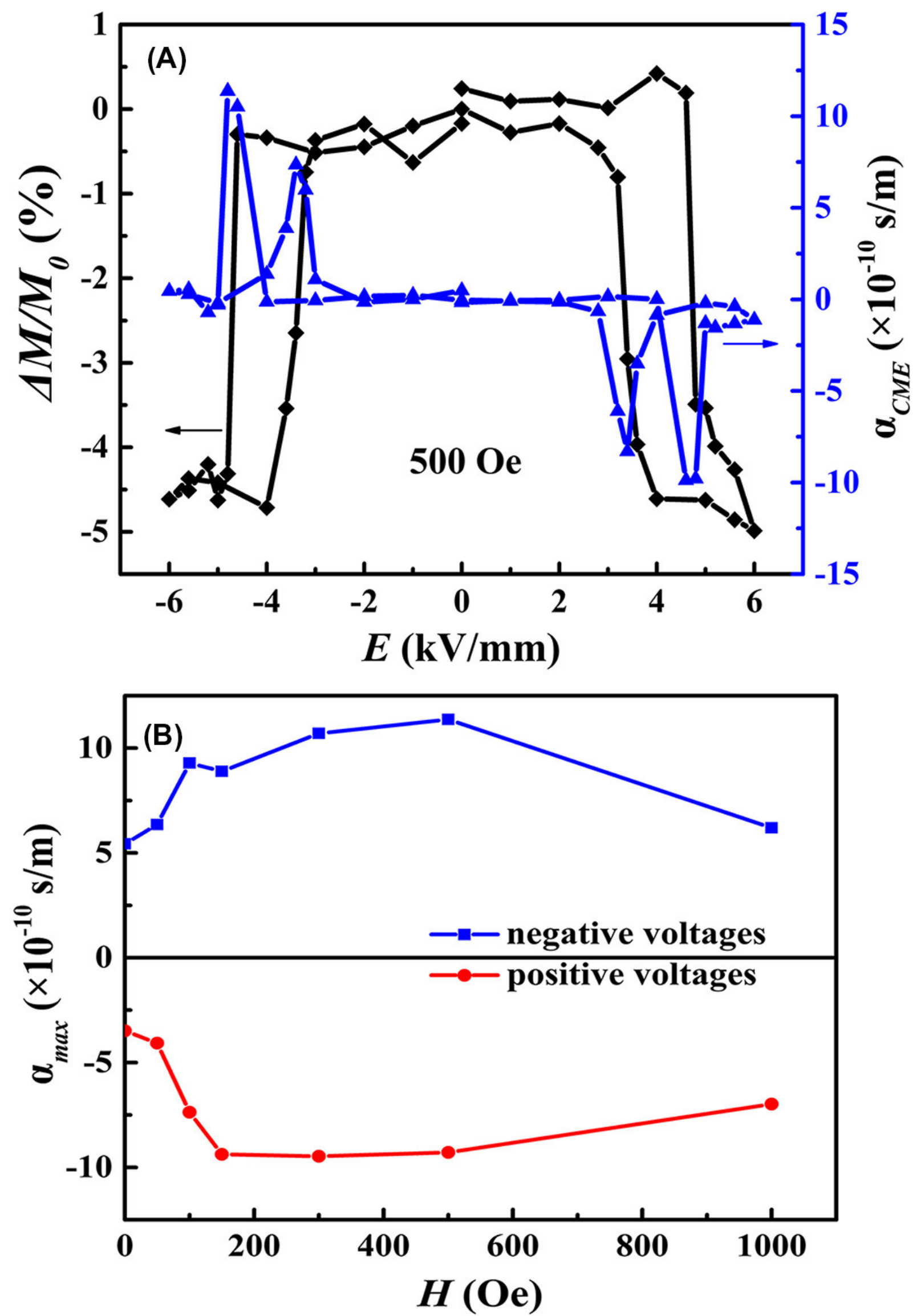

Figure 3: A, $\Delta M / M_{0}$ and CME coefficient vs $E$ under a bias magnetic field of $500 \mathrm{Oe}$; $\mathrm{B}$, the maximum of CME coefficient vs bias $H$ under positive and negative voltages of NMG/PLZST/NMG sample. 
The converse ME coupling coefficient can be calculated using the equation: $\alpha_{\mathrm{CME}}=\mu_{0} \delta M / \delta E$ $\left(\mu_{0}=4 \pi \times 10^{-7} \mathrm{H} / \mathrm{m}\right)$. In Figure $3 \mathrm{~A}$, the relationship between electric field tuning magnetization change and CME coefficient is illustrated. Since the sharp magnetization change occurs at the forward and backward switching fields of PLZST ceramic, four peaks of CME coefficient happen correspondingly to the four switching fields. The peak values of CME coefficient vs bias $H$ curves are given in Figure 3B. The $\left(\Delta \mathrm{M} / \mathrm{M}_{0}\right)-E$ loop is symmetric with $E$, so the $\alpha_{\max }$ shows similar trend under positive and negative fields but different signs according to its definition. A maximum CME coefficient of $1.14 \times 10^{-9} \mathrm{~s} / \mathrm{m}$ is achieved at 500 Oe. Even if the CME coefficient value is not very high, these results clearly demonstrate the practicability of strain-mediated CME coupling effect in ferromagnetic antiferroelectric heterostructure.

\section{Conclusions}

In summary, the converse magnetoelectric effect and the electric field tuning of magnetization based on a composite consisting of NMG films and PLZST antiferroelectric ceramic is investigated in this work. Experimental results show that the AFE-FE phase transition strain induces a strong magnetic anisotropy change in NMG films. It is remarkable that first, the evolution of $\left(\Delta M / M_{0}\right)$ as a function of $E$ is in good agreement with the ceramic strain evolution and secondly that the maximum $\Delta M / M_{0}$ variation, which attains a maximum value of $15 \%$, is reached at $0 \mathrm{Oe}$ (without bias magnetic field). This work which investigates the magnetization evolution with electric field of a FM/AFE composite clearly demonstrates the potentiality of this kind of structure where, at the best of the authors' knowledge, AFE material is used for the first time.

\section{Acknowledgments}

We thank Shanghai Institute of Ceramics and Université Polytechnique Hauts-de-France for the overseas academic exchange.

\section{References}

[1] Klimov A, Tiercelin N, Dusch Y, Giordano S, Mathurin T, Pernod P, et al. Magnetoelectric write and read operations in a stress-mediated multiferroic memory cell. Appl Phys Lett. 2017; 110(22): 222401.

[2] S, Garcia V, Barthelemy A, Bibes M. Magnetoelectric devices for spintronics. Annu Rev Mater Res. 2014; 44(1): 91- 116.

[3] Vopson MM, Zhou D, Caruntu G. Multicaloric effect in bi-layer multiferroic composites. Appl Phys Lett. 2015; 107(18): 182905.

[4] Liu M, Obi O, Lou J, Chen Y, Cai Z, Stoute S, et al. Giant electric field tuning of magnetic properties in multiferroic ferrite/ferroelectric heterostructures. Adv Funct Mater. 2009; 19(11): $1826-31$.

[5] Das J, Song YY, Mo N, Krivosik P, Patton CE. Electric-field-tunable low loss multiferroic ferromagnetic- ferroelectric heterostructures. Adv Mater. 2009; 21(20): 2045-9. 
[6] Liu M, Nan T, Hu JM, Zhao SS, Zhou Z, Wang CY, et al. Electrically controlled nonvolatile switching of magnetism in multiferroic heterostructures via engineered ferroelastic domain states. NPG Asia Mater. 2016; 8(9): e316.

[7] Ederer C, Spaldin NA. Weak ferromagnetism and magnetoelectric coupling in bismuth ferrite. Phys Rev. 2005; 71(6): 060401.

[8] Lawes G, Srinivasan G. Introduction to magnetoelectric coupling and multiferroic films. $J$ Phys D Appl Phys. 2011; 44(24): 243001.

[9] Eerenstein W, Mathur ND, Scott JF. Multiferroic and magnetoelectric materials. Nature. 2006; 442(7104): 759- 65 .

[10] Vaz C, Hoffman J, Ahn CH, Ramesh R. Magnetoelectric coupling effects in multiferroic complex oxide composite structures. Adv Mater. 2010; 22(26-27): 2900- 18.

[11] Islam RA, Priya S. Effect of piezoelectric grain size on magnetoelectric coefficient of $\mathrm{Pb}\left(\mathrm{Zr}_{0.52} \mathrm{Ti}_{0.48}\right) \mathrm{O}_{3}-\mathrm{Ni}_{0.8} \mathrm{Zn}_{0.2} \mathrm{Fe}_{2} \mathrm{O}_{4}$ particulate composites. J Mater Sci. 2008; 43(10): 3560- 8.

[12] Fang Z, Lu SG, Li F, Datta S, Zhang QM, El Tahchi M. Enhancing the magnetoelectric response of Metglas/polyvinylidene fluoride laminates by exploiting the flux concentration effect. Appl Phys Lett. 2009; 95(11): 112903.

[13] Vopson MM. Fundamentals of multiferroic materials and their possible applications. Crit Rev Solid State. 2015; 40(4): 223- 50.

[14] Erdem D, Bingham NS, Heiligtag FJ, Pilet N, Warnicke P, Vaz C, et al. Nanoparticlebased magnetoelectric $\mathrm{BaTiO}_{3}-\mathrm{CoFe}_{2} \mathrm{O}_{4}$ thin film heterostructures for voltage control of magnetism. ACS Nano. 2016; 10(11): 9840- 51.

[15] Guo Y, Liu Y, Withers RL, Brink F, Chen H. Large electric field-induced strain and antiferroelectric behavior in $(1-x)\left(\mathrm{Na}_{0.5} \mathrm{Bi}_{0.5}\right) \mathrm{TiO}_{3}-x \mathrm{BaTiO}_{3}$ ceramics. Chem Mater. 2011; 23(2): $219-28$.

[16] Lian J, Ponchel F, Tiercelin N, Chen Y, Rémiens D, Lasri T, et al. Electric field tuning of magnetism in heterostructure of yttrium iron garnet film/lead magnesium niobate-lead zirconate titanate ceramic. Appl Phys Lett. 2018; 112(16): 162904.

[17] Li G, Liu Y. Stress-induced change of magnetization in a Ni-Mn-Ga single crystal under magnetomechanical training. Appl Phys Lett. 2006; 88(23): 232504.

[18] Figueiras F, Rauwel E, Amaral VS, Vyshatko N, Kholkin AL, Soyer C, et al. Study of $\mathrm{Ni}_{2}-$ $\mathrm{Mn}-\mathrm{Ga}$ phase formation by magnetron sputtering film deposition at low temperature onto $\mathrm{Si}$ substrates and $\mathrm{LaNiO}_{3} \mathrm{~Pb}(\mathrm{Ti}, \mathrm{Zr})_{\mathrm{O}} 3$ buffer. J Vac Sci Technol A. 2009; 28(1): 6- 10.

[19] Niazi A, Poddar P, Rastogi AK. A precision, low-cost vibrating sample magnetometer. Curr Sci. 2000; 79(1): 11.

[20] Rondinelli JM, Stengel M, Spaldin NA. Carrier-mediated magnetoelectricity in complex oxide heterostructures. Nat Nanotechnol. 2008; 3(1): 46- 50. 